\title{
Évolution de la normalisation dans le domaine des oléagineux et des corps gras
}

Oléagineux, Corps Gras, Lipides. Volume 10, Numéro 4, 256-9, JUILLET-AOÛT 2003, Méthodologie

Auteur(s) : Alain QUINSAC, Service Valorisation, Qualité et Sécurité sanitaire, CETIOM, Pessac 33600 France Tel : 0556073084 Fax : 0556079718 <quinsaccetiom.fr>.

Author(s) : Alain QUINSAC

Résumé : La normalisation joue un grand rôle dans les échanges économiques en participant à l'ouverture et à la transparence des marchés. La filière des Oléagineux et des Corps Gras a intégré depuis longtemps la normalisation dans sa stratégie. Élaborés à partir des besoins de la profession et notamment au niveau de la relation client-fournisseur, les programmes ont concerné principalement l'échantillonnage et l'analyse. Depuis quelques années, une forte évolution du contexte socioéconomique et réglementaire (utilisation non-alimentaire, sécurité alimentaire, assurance qualité, ) a élargi le champ de la normalisation. La démarche normative adoptée dans le cas des bio-diesels et de la détection des OGM dans les oléagineux est expliquée. Les conséquences de l'évolution de la normalisation et les enjeux pour la profession des oléagineux dans le futur sont évoqués.

Summary : Standardization is essential in trading while taking part in the opening and the transparency of the markets. The French organization of oilseeds and fats has integrated standardization in its strategy. The programs mainly relate to sampling and analysis using methods adapted to the needs for the profession at customer-supplier level. For a few years a strong evolution of the socio-economic and regulatory context (non food use, food safety, quality insurance) has widened the field of standardization. The standardization processes in the cases of bio-diesel and GMO detection standardization are described. Consequences and stakes of the standardization trend concerning the oilseeds French Organization are recalled.

Mots-clés : Normalisation, oléagineux, corps gras, analyse

Keywords : Standardization, oilseeds, fats, analysis

\section{ARTICLE}

Auteur(s) : Alain QUINSAC

Service Valorisation, Qualité et Sécurité sanitaire, CETIOM, Pessac 33600 France

Tel : 0556073084

Fax : 0556079718

quinsac@cetiom.fr 


\section{De la réglementation...}

Initiée vers la fin du XIX ${ }^{e}$ siècle par les pouvoirs publics pour le contrôle des denrées alimentaires, la normalisation dans le secteur agroalimentaire était surtout le fait d'une commission centrale composée de chimistes de la répression des fraudes et de professeurs renommés [1]. Les méthodes officielles qui ont été élaborées ont constitué la base de l'arsenal des méthodes que nous connaissons aujourd'hui. Entre les deux guerres, l'évolution du commerce a de plus en plus conduit les industriels, les importateurs et exportateurs à pratiquer le contrôle des marchandises et à s'impliquer dans la démarche normative. Ce mouvement s'est fortement accentué après 1945 et s'est traduit par la création de plusieurs organismes de normalisation internationaux: I'ISO (International Standardization Organisation) en liaison avec les organismes nationaux, I'AFNOR (Association française de normalisation) en France, le DIN (Deutsches Institut fr Normung) en Allemagne, le BSI (British Standards Institution) au Royaume-Uni, etc... Plus récemment, en 1961, a été créé le CEN (Comité Européen de Normalisation) pour accompagner la construction européenne. Ces organismes ont un champ d'activité très large, couvrant une grande gamme de produits agricoles ou industriels et de services. Ils ont mis en place des règles de fonctionnement et de collaboration qui assurent une certaine cohérence entre les normes adoptées et les travaux programmés à l'échelon national, européen ou mondial. Cet esprit de coopération n'est cependant pas aussi largement répandu qu'il le faudrait dans le monde de la normalisation. Des désaccords existent encore entre les méthodes issues de ces organismes et certaines méthodes officielles nationales, ou émanant d'organismes internationaux tels que I'AOAC International (Association of Analytical Communities) ou l'AOCS (American Oil Chemist's Society) ayant acquis depuis longtemps une grande notoriété et qui ont donc l'avantage de l'antériorité et d'être parfaitement reconnus par les "traders" anglo-saxons. Ainsi la FOSFA (Federation of Oils, Seeds and Fats Associations) utilise exclusivement pour ses transactions de graines oléagineuses la méthode AOCS de détermination de la teneur en huile, au détriment de la méthode ISO. Entre les deux méthodes, l'écart de résultat sur la teneur en huile est très faible, mais appliqué sur de grandes quantités de graines, il finit par représenter des sommes importantes qui deviennent des sources de conflit. La "nouvelle approche " européenne appliquée depuis une dizaine d'années a pour but à long terme d'homogénéiser les réglementations, mais provoque parfois des distorsions de concurrence. Elle consiste à reconnaître conformes en France des produits originaires de I'Union Européenne (UE) qui respectent les normes en vigueur dans leur pays d'origine, mais qui ne le seraient pas s'ils étaient produits en France avec une réglementation française plus stricte [2]. De ce fait, des producteurs nationaux peuvent être moins concurrentiels que des producteurs étrangers.

\section{... à la démarche volontaire}

Les industriels, pour ne pas être défavorisés, ont intérêt à prendre à leur compte les actions de normalisation et à les intégrer comme un vecteur de performances dans leurs activités et stratégies de production. La normalisation joue désormais un grand rôle dans les échanges économiques en participant à l'ouverture, à la transparence et à la moralisation du marché. Les normes permettent le maintien ou l'amélioration de la qualité des produits en donnant aux parties prenantes (clientsfournisseurs) les éléments de référence et le langage commun nécessaires à l'établissement des contrats. Dans le cas notamment d'entreprises certifiées, la norme est devenue un moyen de négociation et un instrument de relations. Elle peut être également un outil de différenciation : la norme de spécification, en créant une segmentation du marché, dynamise celui-ci car le 
positionnement de nouveaux produits est alors possible. II faut noter que des spécifications sont souvent définies dans le cadre de contrats commerciaux. L'huile de tournesol « oléique » répondant à une spécification nouvelle concernant la teneur en acide oléique a permis de créer un marché nouveau dans les secteurs alimentaire et non alimentaire. Le succès des labels dans l'agroalimentaire est également une illustration courante de l'efficacité de la spécification des produits en vue de leur identification et différenciation par le consommateur. Le processus d'élaboration des normes, avec la prise en compte du besoin, les études techniques, la recherche et l'obtention d'un consensus, nécessite un investissement important en temps et en travail de la part des acteurs impliqués. Les délais peuvent paraître élevés car on compte souvent plusieurs années entre le besoin exprimé et la publication. Très récemment, le CEN dans son "New agenda " [3] a d'ailleurs réduit les délais d'élaboration de 68 à 36 mois en jouant principalement sur les travaux techniques, les délais d'enquête et de traduction étant maintenus. Cela va signifier pour l'avenir que des travaux de normalisation ne seront engagés que si la mise au point technique de la méthode dans le domaine d'application visé est réalisée. Les études restant à accomplir se concentreront alors sur la rédaction, la prise en compte des exigences métrologiques, les circuits inter-laboratoires et les calculs statistiques, la focalisation des moyens sur ces études devant se traduire par une meilleure validation de la méthode. Un autre objectif de ce nouvel agenda avec la réduction des délais est évidemment d'éviter de répondre à un besoin qui a évolué depuis ou qui a disparu.

\section{L'exemple de la filière Oléagineux et Corps Gras}

Depuis quelques années, les enjeux de la normalisation ont pris une importance considérable dans ce secteur en pleine évolution. La libéralisation et la mondialisation des échanges, les exigences de qualité, de sécurité sanitaire, de traçabilité requièrent des normes "horizontales " couvrant des domaines de plus en plus larges, c'est-à-dire applicables à plusieurs types de produits (oléagineux, céréales, plats cuisinés, etc) comme par exemple, la détection des OGM. D'autre part, les méthodes d'analyse doivent être capables de répondre à des besoins toujours croissants en matière de spécificité, limite de détection, fidélité et justesse. La filière des Oléagineux et des Corps Gras avec le soutien de I'ONIDOL (Organisation Nationale Interprofessionnelle des Oléagineux), de la FNCG (Fédération Nationale des industries des Corps Gras), de I'ITERG (Institut des Corps Gras) et du CETIOM (Centre Technique Interprofessionnel des Oléagineux Métropolitains), a depuis longtemps intégré la normalisation dans sa stratégie. Les programmes ont concerné principalement l'analyse, avec la mise en place de méthodes communes adaptées aux besoins de la profession au niveau de la relation client-fournisseur. La prise en compte du contexte socio-économique et réglementaire a élargi le champ de la normalisation. Des instances de réflexion, de programmation et d'élaboration ont été créées et des domaines entièrement nouveaux, pris en compte (utilisation non-alimentaire, sécurité alimentaire, traçabilité, accréditation des laboratoires, certification, ...). Pour toute méthode normalisée, il est désormais nécessaire de considérer les nouvelles exigences des référentiels de qualité adoptés par les laboratoires. La métrologie, les matériaux de référence, les incertitudes, les performances de fidélité font partie intégrante de la norme et participent à sa valeur opérationnelle. L'élargissement du domaine d'application des normes ou l'apparition de besoins nouveaux peut être illustré par les exemples de la détection des OGM (organismes génétiquement modifiés) et les biodiesels. 


\section{La détection des OGM}

Suite à l'arrivée du soja génétiquement modifié RRS (Round-Up Ready Soybean) sur les marchés français et européen et pour satisfaire à la demande sociétale en matière de sécurité sanitaire et de traçabilité, l'administration a initié des travaux de normalisation sous l'égide du CEN/TC275 et de I'AFNOR (commission V03E) avec la participation active de laboratoires officiels (INRA, GEVES, DGCCRF) et de nombreux autres acteurs concernés: laboratoires prestataires de services, semenciers, instituts, associations de consommateurs, industriels de l'agroalimentaire, etc. Tout étant à construire, les premiers travaux ont consisté à élaborer une norme horizontale, c'est-à-dire à définir, à l'usage des laboratoires, les lignes directrices des bonnes pratiques d'analyse et de détection des OGM dans les produits alimentaires. Plusieurs facteurs ont rendu cet objectif difficile à atteindre dans le bref délai souhaité. Tout d'abord, la technique PCR (polymerase chain reaction) utilisée, assez récente, a évolué rapidement tant au niveau des appareillages que des systèmes réactionnels, et s'est heurtée à une contrainte assez nouvelle dans le domaine de l'analyse : les événements, séquences ou parties de gène que la technique est censée détecter ne sont pas toujours rendus publics par les fabricants d'OGM. Les performances des analyses PCR s'avéraient donc hasardeuses d'autant qu'il était demandé de détecter une présence d'OGM à un niveau très bas dans pratiquement tout produit agroalimentaire, de la semence au plat cuisiné. L'absence de références techniques communes aux membres de la commission sur les performances des méthodes a également constitué un handicap: comment par exemple, définir une limite de détection si l'on ne connaît pas la répétabilité de la méthode. Enfin, la normalisation fut victime de son succès, le nombre élevé de participants d'horizons très différents et débutant dans la démarche normative a engendré des débats riches mais parfois confus. Ces travaux ont été concrétisés en décembre 2000 par la publication de la norme expérimentale XP VO3-020-1. Cette norme a largement inspiré le projet de norme prEN ISO 24276 traitant du même sujet et élaboré par le CEN/TC 275. Au niveau français, la norme est actuellement en cours de révision et verra son contenu relatif à la technique PCR qualitative et quantitative traité dans une norme spécifique dont la publication est prévue en 2004. Les aspects échantillonnage et extraction de I'ADN sont étudiés au niveau européen avec des publications prévues respectivement en 2005-2006 et 2004. Les normes européennes sur la PCR qualitative et quantitative sont respectivement prévues pour 2004 et 20052006.

En parallèle, la filière oléagineuse française a souhaité en 1999 mener des travaux davantage ciblés dans un but d'efficacité et de rapidité. Par l'intermédiaire de la commission AFNOR/T60D « Graines oléagineuses et tourteaux ", elle a obtenu la validation de cette demande au comité technique homologue de I'ISO (TC34/SC2) qui I'a retransmise à I'ISO/TC34 et au CEN/TC 275 en charge des méthodes horizontales et du dossier « détection OGM ». Cette procédure est rendue obligatoire par des accords dits " de Vienne " qui ont pour objectif d'éviter la duplication inutile de travaux de normalisation à l'ISO et au CEN. L'AFNOR a donc été autorisée à créer un groupe "Oléagineux » en mars 2001 pour travailler sur un sujet bien précis : I'analyse quantitative de l'OGM « Round-up-Ready Soybean " dans les graines de soja. La focalisation du domaine d'application qui représente néanmoins la majorité des analyses à l'import des graines oléagineuses en France, avait pour but de simplifier considérablement les études et d'envisager la réalisation d'un circuit inter-laboratoires dans des délais assez courts. De ce fait, un groupe de sept à huit laboratoires très actifs s'est 
constitué et a permis d'obtenir très rapidement des données de fidélité des méthodes PCR les plus largement utilisées en France. Ces données ont d'ailleurs permis à la commission AFNOR, détection des OGM (VO3E), de réfléchir plus concrètement sur les notions de limite de détection et de quantification et de travailler sur des niveaux plus réalistes. Le groupe "Oléagineux » a poursuivi ses travaux par des comparaisons de méthodes et de kits d'extraction et purification de I'ADN dans le cadre d'un autre circuit inter-laboratoires. La prise en compte des travaux du groupe " oléagineux " AFNOR par le groupe de travail créé à l'ISO (ISO/TC34/WG7) a été réalisée en octobre 2001. En juin 2003, une proposition de norme verticale décrivant la quantification de l'OGM « Round-Up Ready Soybean " est élaborée. Ce texte va maintenant entrer dans le processus classique de validation des normes internationales, qui comprend deux votes techniques et deux votes éditoriaux ainsi que la validation de la méthode décrite par un essai inter-laboratoire international. La publication de cette norme par I'ISO est prévue pour 2005 ou 2006. Cet exemple, portant sur un sujet particulièrement délicat, a montré que la normalisation a été particulièrement active. D'abord sous l'impulsion des pouvoirs publics, puis de l'interprofession des oléagineux, les travaux ont rassemblé la majorité des acteurs de la filière traditionnelle ainsi que d'autres habituellement moins impliqués dans ce type de démarche tels que les consommateurs. Les organismes normatifs nationaux et internationaux ont pris en compte les besoins et organisé les relations entre les équipes en conséquence. À cet égard, il faut souligner l'influence des comités techniques du CEN et de I'ISO. La France, qui en assure les secrétariats (CEN/TC307 et ISO/TC34/SC2) avec le relais de I'AFNOR, a réussi à faire partager aux niveaux européen et international les préoccupations de l'interprofession des oléagineux et ainsi, à mobiliser fortement les compétences vers l'objectif visé. De manière générale, la normalisation dans le domaine de la détection des OGM a constitué un vecteur d'information entre les différents acteurs et a fréquemment contribué à l'établissement d'un consensus. Les bio-diesels ont constitué depuis quelques années un sujet particulièrement lourd, tant pour la mise au point de méthodes entièrement nouvelles que pour la prise en compte de spécifications d'origine industrielle et donc inhabituelles dans le domaine de l'alimentaire. À la demande des utilisateurs des biocarburants (motoristes et surtout pétroliers), la Commission Européenne (DG XII Energie) a mandaté le CEN en 1997 pour élaborer des normes d'analyse et de spécification [4]. Le mandat (M/245) pris en charge par les deux comités techniques concernés, le CEN/TC19 (Produits pétroliers, lubrifiants et produits dérivés) et le CEN/TC307 (Graines oléagineuses et corps gras), a permis la mise au point d'une dizaine de méthodes nouvelles, applicables à l'analyse des bio-diesels de manière à garantir leur utilisation sans risque. Les travaux de normalisation ont été répartis dans trois groupes de travail composés d'experts de différentes filières pour prendre en compte la diversité des besoins. Un quatrième groupe représentatif des comités techniques TC307 et TC19 était chargé de la coordination. L'essentiel des études analytiques a été réalisé par I'ITERG. Dix normes sont actuellement publiées (NF EN 14103 à NF EN 14114). Cinq autres normes sont actuellement en cours de ratification au CEN et seront ensuite reprises au niveau national. Ces documents vont servir d'outils pour l'application de la directive JOUE $\mathrm{n}^{\circ}$ L. 123 visant à promouvoir l'utilisation des biocarburants et fixant aux États membres des objectifs minimum de $2 \%$ de parts de marché en 2005 et 5,75\% en 2010. Cet effort de normalisation n'est pas définitif. Déjà, les utilisateurs font état de besoins nouveaux. L'évolution des moteurs diesel et l'origine de plus en plus diversifiée des huiles végétales imposent des exigences plus grandes sur la précision des méthodes existantes et entraînent la prise en compte de spécifications nouvelles. Le financement de ces nouveaux travaux de mise au point n'est pas encore arrêté, il devra se faire à l'échelle de l'Europe. La normalisation dans le domaine bio-diesel a été exclusivement une affaire européenne. Dans le domaine des graines oléagineuses et des corps 
gras, c'est le seul cas où le CEN ait été entièrement à l'origine de la demande et de la réalisation de normes. À ce titre, cela demeure un exemple réussi de l'élaboration d'un outil normatif destiné à favoriser la promotion et le développement d'un produit et d'une filière à l'échelle européenne.

\section{En conclusion}

La normalisation n'est pas seulement une affaire de spécialistes. Au côté des experts techniques, une participation multidisciplinaire représentative des utilisateurs est plus que jamais nécessaire pour initier des travaux, équilibrer les débats et faire en sorte que les normes produites soient des outils utiles, adaptés, consensuels, fiables et efficients. Le bénéfice collectif ou individuel de la norme n'est malheureusement pas suffisamment perçu ou alors par défaut lorsqu'il y a carence. Au niveau du commerce international, l'utilisation des normes se traduit parfois par une confrontation entre deux référentiels servant différemment les intérêts des parties prenantes (client-fournisseur). Les échanges, les relations entre les organisations de normalisation (ISO, CEN) et de commerce (FOSFA) et l'harmonisation des normes doivent à terme réduire ces difficultés. La mise aux point de normes transversales est également nécessaire pour simplifier et réduire les coûts. Dans cet objectif, la défense des intérêts nationaux et professionnels est nécessaire et sera conditionnée par la vigueur et la représentativité de l'activité de la normalisation dans le cadre national et international avec le soutien de l'AFNOR.

1. Commissions et comités techniques impliqués dans la normalisation des oléagineux et corps gras en France, au CEN et à I'ISO.

\begin{tabular}{l|l|l|l|}
\hline Domaine d'application & Secrétariat & Présidence & \\
\hline AFNOR/T60 C & $\begin{array}{l}\text { Corps gras d'origine animale et } \\
\text { végétale et dérivés }\end{array}$ & AFNOR & ITERG \\
\hline AFNOR/T60 D & Graines oléagineuses et tourteaux & AFNOR & CETIOM \\
\hline AFNOR/V03 E & $\begin{array}{l}\text { Produits agroalimentaires. } \\
\text { Détection des OGM }\end{array}$ & AFNOR & LIMAGRAIN \\
\hline CEN/TC275 & $\begin{array}{l}\text { Produits agroalimentaires. } \\
\text { Méthodes horizontales }\end{array}$ & $\begin{array}{l}\text { Allemagne } \\
\text { (DIN) }\end{array}$ & Allemagne \\
\hline CEN/TC307 & $\begin{array}{l}\text { Graines oléagineuses. Corps gras } \\
\text { d'origine animale et végétale et } \\
\text { dérivés }\end{array}$ & $\begin{array}{l}\text { France } \\
\text { (AFNOR) }\end{array}$ & France \\
\hline CEN/TC19 & $\begin{array}{l}\text { Produits pétroliers, lubrifiants et } \\
\text { produits dérivés }\end{array}$ & $\begin{array}{l}\text { Pays-Bas } \\
\text { (NEN) }\end{array}$ & Pays-Bas \\
\hline ISO/TC/34/SC2 & $\begin{array}{l}\text { Graines et fruits oléagineux et } \\
\text { tourteaux de graines oléagineuses }\end{array}$ & $\begin{array}{l}\text { France } \\
\text { (AFNOR) }\end{array}$ & France \\
\hline
\end{tabular}




\begin{tabular}{|c|c|c|c|}
\hline ISO/TC/34/SC11 & $\begin{array}{l}\text { Corps gras d'origine végétale et } \\
\text { animale et dérivés }\end{array}$ & $\begin{array}{l}\text { Royaume-Uni } \\
\text { (BSI) }\end{array}$ & Allemagne \\
\hline ISO/TC/34/WG7 & Détection des OGM & $\begin{array}{l}\text { France } \\
\text { (AFNOR) }\end{array}$ & France \\
\hline
\end{tabular}

L'auteur remercie Florence LACOSTE (ITERG-Pessac), responsable du groupe de travail CEN/TC307/WG1 sur les bio-diesels, Emmanuelle PIC (CETIOM-Thiverval-Grignon), responsable du groupe "Oléagineux » au sein de la commission AFNOR/VO3E pour leur contribution. Claudine Muzet et Carole Moretain de l'AFNOR, en charge du suivi de la normalisation dans le domaine Oléagineux et Corps gras. Les travaux des commissions ISO/TC34/SC2 et CEN/TC307 sont consultables aux adresses respectives suivantes : http://comelec.afnor.fr/iso/tc34sc2

et http://comelec.afnor.fr/cen/tc307.

\section{RÉFÉRENCES}

1. WOLFF J-P. Méthodes d'analyses et normalisation. OCL, $1995 ; 2$ : 112-113.

2. NORMAND N. Spécificité de la normalisation en agroalimentaire. OCL, $1995 ; 2$ : 116-120.

3. AFNOR (2003). Compte-rendu de la $7^{\mathrm{e}}$ réunion du CEN/TC307. Berlin 15, 16 mai 2003. AFNOR/T60D/Doc N 113, Annexe 1.

4. MUZET-MICONNET C (2003). Biocarburants : directive et normes arrivent. Enjeux, Eds Afnor, Saint Denis La Plaine, $\mathrm{n}^{\circ} 237: 18 . \mathrm{N}$

Entité 